\title{
Association of Gc-globulin variation with susceptibility to COPD and diffuse panbronchiolitis
}

\author{
T. Ishii*, N. Keicho\#, S. Teramoto ${ }^{\star}$, A. Azuma ${ }^{+}$, S. Kudoh ${ }^{+}$, Y. Fukuchi ${ }^{\S}$, Y. Ouchi*, T. Matsuse ${ }^{f}$
}

Association of Gc-globulin variation with susceptibility to COPD and diffuse panbronchiolitis. T. Ishii, N. Keicho, S. Teramoto, A. Azuma, S. Kudoh, Y. Fukuchi, Y. Ouchi, T. Matsuse. C ERS Journals Ltd 2001.

ABSTRACT: Chronic obstructive pulmonary disease (COPD) and diffuse panbronchiolitis (DPB) are both characterized by chronic airflow limitation. Although the aetiology of these diseases is under investigation, it is commonly hypothesized that neutrophils have a major role in the disease pathogenesis. The variation of the genes related to chemotaxis of neutrophils may confer a risk for the development of both COPD and DPB.

In the present report, the authors investigated the association between genetic variation that codes for the 416th and 420th amino acid of Gc-globulin, reported to be associated with chemotaxis of neutrophils, and susceptibility to COPD and DPB. Blood samples obtained from patients with COPD $(n=63)$, DPB $(n=82)$, and control subjects $(n=82)$ were used for the genotyping assay.

The proportion of $G C^{*} 1 \mathrm{~F}$ homozygotes was significantly higher in the COPD patients than the control subjects (COPD $36.5 \%$ versus control $20.7 \%$ ), and the odds ratio for $\mathrm{GC} * 1 \mathrm{~F}$ homozygotes was $2.2(95 \%$ confidence interval $1.1-4.6)$ for the COPD group. There was no difference on the distribution of the other genotypes $(\mathrm{GC} * 1 \mathrm{~F}-1 \mathrm{~S}$ heterozygotes, GC*1S homozygotes, GC*2-1F heterozygotes, GC*2-1S heterozygotes and $\mathbf{G C} * 2$ homozygotes) or the allele frequencies among these groups.

These findings suggest that the GC*1F gene polymorphism of Gc-globulin may be one of the risk factors for chronic obstructive pulmonary disease. However, no association between this polymorphism of Gc-globulin and susceptibility to diffuse panbronchiolitis was found.

Eur Respir J 2001; 18: 753-757.

\begin{abstract}
*Dept of Geriatric Medicine, University of Tokyo, Tokyo; " Dept of Respiratory Diseases, Research Institute, International Medical Center of Japan, Tokyo; "Dept of Internal Medicine, San-no Hospital, and Medical Research Center, International University of Health and Welfare, Tokyo; ${ }^{+}$Fourth Dept of Internal Medicine, Nippon Medical School, Tokyo; ${ }^{8}$ Dept of Respiratory Medicine, Juntendo University School of Medicine, Tokyo; and ${ }^{f}$ Dept of Pulmonary Medicine, Yokohama City University Medical Center, Yokohama, Japan.
\end{abstract}

Correspondence: T. Matsuse, Dept of Pulmonary Medicine, Yokohama City University Medical Center, 4-57, Urahune-cho, Minami-ku, Yokohama City, 232-0024 Japan.

Fax: 81452412812

Keywords: Chronic obstructive pulmonary disease, diffuse panbronchiolitis, Gc-globulin (vitamin D-binding protein), genetic variation

Received: November 292000

Accepted after revision June 252001

This study was partly supported by a grant from the Smoking Research Foundation, Health Sciences Research Grants from Ministry of Health and Welfare, and Grant-in-Aid for Scientific Research from the Ministry of Education, Science, Sports and Culture, Japan.
Chronic obstructive pulmonary disease (COPD) is now becoming the most prevalent respiratory disease, and it is estimated that COPD will be the fifth leading cause of the Disability-Adjusted Life Year in the world in 2020 [1]. Although the most prevalent risk factor for the development of COPD is tobacco smoking, only $10-20 \%$ of heavy smokers develop symptomatic COPD [2]. Recent studies suggest that genetic factors, excluding $\alpha 1$-antitrypsin, might be related to the individual susceptibility to COPD [3, 4]. Additionally, based on the protease-antiprotease hypothesis on the pathogenesis of COPD [5], neutrophils that produce neutrophil elastase have been thought to play a key role in the pathogenesis of COPD.

Diffuse panbronchiolitis (DPB), first described in 1969 [6], is also characterized by chronic airflow limitation, [6], and the aetiology of the disease is still unknown. Cases of DPB are mainly found in East Asian populations [7, 8], and neither environmental factors nor infections specific to the disease have been demonstrated. It has also been reported that human leukocyte antigen-B alleles or a closely linked gene contribute to genetic predisposition in DPB [9]. After considering these facts, the authors hypothesized that genetic factors may be associated with the pathogenesis of DPB. The chemotactic activity of neutrophils is considered to be important in the pathogenesis of DPB because: 1) DPB is characterized by the infiltration of inflammatory leukocytes into the respiratory bronchioles; and 2) erythromycin, the first choice of therapy for DPB, was reported to inhibit the elastolytic-like activity [10] and chemotactic activity of neutrophils [10-12].

Gc-globulin (vitamin D-binding protein) is known 
to bind with vitamin D and transport it. However, it has also been reported that Gc-globulin enhances the neutrophil chemotactic activity of complement component 5a (C5a), C5a without the carboxylterminal arginine (C5a des Arg) [13] and human monocyte chemotaxis by a different pathway [14]. Gc-globulin exhibits considerable genetic variability due to its three polymorphic alleles $\left(\mathrm{GC}^{*} 1 \mathrm{~F}, \mathrm{GC}^{*} 1 \mathrm{~S}\right.$, and $\left.\mathrm{GC}^{*} 2\right)$ and $>80$ rarer alleles [15]. Although several investigators have previously reported an association between this genetic variation and COPD, the conclusion is controversial [16-19]. Low frequency for the $\mathrm{GC}^{*} 2 / \mathrm{GC}^{*} 2$ genotype in COPD was observed in two reports at a serological level [16, 17], and in one report at a deoxyribonucleic acid (DNA) level [18]. However, KAUFFMANN et al. [19] did not detect any association between this genotype and COPD. Although Horne et al. [17] reported a positive association between $\mathrm{GC}^{*} 1 \mathrm{~F}$ allele and airway obstruction, others have not yet been able to confirm this result $[16,18,19]$.

In this study, the authors investigated whether the three polymorphic alleles $\left(\mathrm{GC}^{*} 1 \mathrm{~F}, \mathrm{GC}^{*} 1 \mathrm{~S}\right.$, and $\mathrm{GC}^{* 2}$ ) of Gc-globulin are associated with the susceptibility of COPD and DPB by using a polymerase chain reaction (PCR)-based genotyping assay.

\section{Material and methods}

\section{Study subjects}

The COPD group consisted of 63 adults, who were diagnosed as COPD at the Tokyo University Hospital and the Juntendo University Hospital, Tokyo, Japan according to the guidelines of the American Thoracic Society [20]. The diagnosis was based on the signs and symptoms, as well as on the results of pulmonary function tests including: 1) the percentage of predicted forced expiratory volume in one second (FEV1 \% pred); 2) the residual volume divided by the total lung capacity (RV/TLC); 3) the diffusing capacity of the lung for carbon monoxide ( $D \mathrm{~L}, \mathrm{CO})$ taken with patients in a stable condition (not at the time of exacerbation of the disease); and 4) chest radiographs (including chest computed tomography (CT)). The specific enrolment criteria were: 1) FEV1/forced vital capacity (FVC) ratio $<70 \%$; and 2 ) a smoking history of $\geqslant 10$ pack-yrs. There were no asthma patients in the COPD group. The serum level of $\alpha 1$-antitrypsin for all subjects in the COPD group was within a normal range (mean \pm SEM was $254.0 \pm 7.4 \mathrm{mg} \cdot \mathrm{dL}^{-1}$, range: $159-391$ ). Blood samples of the DPB cases were obtained from the Toranomon Hospital, Tenri Hospital, or Nippon Medical School Hospital, Japan. The diagnostic criteria for DPB proposed in 1995 by a working groups [9] were: 1) persistent cough, sputum, and exertional dyspnoea; 2) past or present history of chronic sinusitis; 3) bilateral diffuse small nodular shadows on a plain chest radiograph film or centrilobular micronodules on chest CT images; 4) coarse crackles; 5) $\mathrm{FEV} 1 / \mathrm{FVC}<70 \%$ and oxygen tension in arterial blood $<10.64 \mathrm{kPa}(<80 \mathrm{mmHg})$; and 6) titre of cold haemagglutinin $\leqslant 64$. A total of $82 \mathrm{DPB}$ patients were recruited for this study and fulfilled criteria 1),2), 3) and at least two of criteria 4), 5), and $6)$. The normal control subjects comprised 82 healthy volunteers from the same area as the DPB cases in Japan. Thus, the subjects of the COPD, DPB and control groups were all Japanese and ethnically matched. The age of the control subjects was not known because they were anonymous donors.

\section{Methods for genotyping}

The method used for genotyping Gc-globulin was modified from a previously described method [18]. Briefly, genomic DNA was extracted from whole blood using the QIAamp Blood Kit (QIAGEN Inc., Valencia, CA, USA). PCR and restriction enzyme fragment length polymorphism assays were performed to genotype the three polymorphic alleles $\left(\mathrm{GC}^{*} 1 \mathrm{~F}\right.$, $\mathrm{GC}^{*} 1 \mathrm{~S}$, and $\mathrm{GC}^{*} 2$ ) of Gc-globulin.

The primer pair Gc-F1: 5'-TAATGAGCAAATG AAAGAAG-3' and Gc-R1: 5'-AATCACAGTAAA GAGGAGGT-3' were used for this assay. PCRs were carried out with a Programme-temp-control System PC-800 PCR machine (Astec, Tokyo, Japan). Genomic DNA (200 ng) was added to a $30-\mu \mathrm{L}$ reaction mixture also containing 22 pmol of each primer and $1 \mathrm{U}$ of AmpliTaq Gold (Perkin-Elmer Applied Biosystems Division, Foster City, CA, USA), in $1 \times$ PCR buffer $(10 \mathrm{mM}$ Tris- $\mathrm{HCl}, 50 \mathrm{mM} \mathrm{KCl}$, $1.5 \mathrm{mM} \mathrm{MgCl} 2,0.001 \%$ (per cent weight in volume $(w / v)$ ) gelatin and $0.2 \mathrm{mM}$ of each deoxynucleotide triphosphate (dNTP). The "auto-hot start" was used to prevent nonspecific priming in the first cycle of PCR. Following an initial denaturation step at $95^{\circ} \mathrm{C}$ for $12 \mathrm{~min}, 40$ cycles of PCR were carried out (denaturation at $95^{\circ} \mathrm{C}$ for $30 \mathrm{~s}$, annealing at $50^{\circ} \mathrm{C}$ for $30 \mathrm{~s}$, elongation at $72^{\circ} \mathrm{C}$ for $60 \mathrm{~s}$ ), which were followed by one cycle of elongation at $72^{\circ} \mathrm{C}$ for $5 \mathrm{~min}$. The PCR products were separated and each was digested with $10 \mathrm{U}$ of Hae III (New England BioLabs Inc., Beverly, MA, USA) and $10 \mathrm{U}$ of Sty I (New England BioLabs Inc.).

All the digests were electrophoresed on a gel containing 2.5\% Ultrapure Agarose (GibcoBRL, Gaithersburg, MD, USA) and ethidium bromide. The film of the bands electrophoresed on the gel is shown in figure 1 .

\section{Data analysis}

The differences in allele distribution and allele frequency among the groups were examined by the Chi-squared test and with the Fisher exact test when appropriate. p-Values $<0.05$ were considered to be statistically significant. All values are expressed as mean \pm SEM.

\section{Results}

The basic characteristics of the COPD and DPB groups were as follows: age (yr) was $68.3 \pm 9.9$ versus 


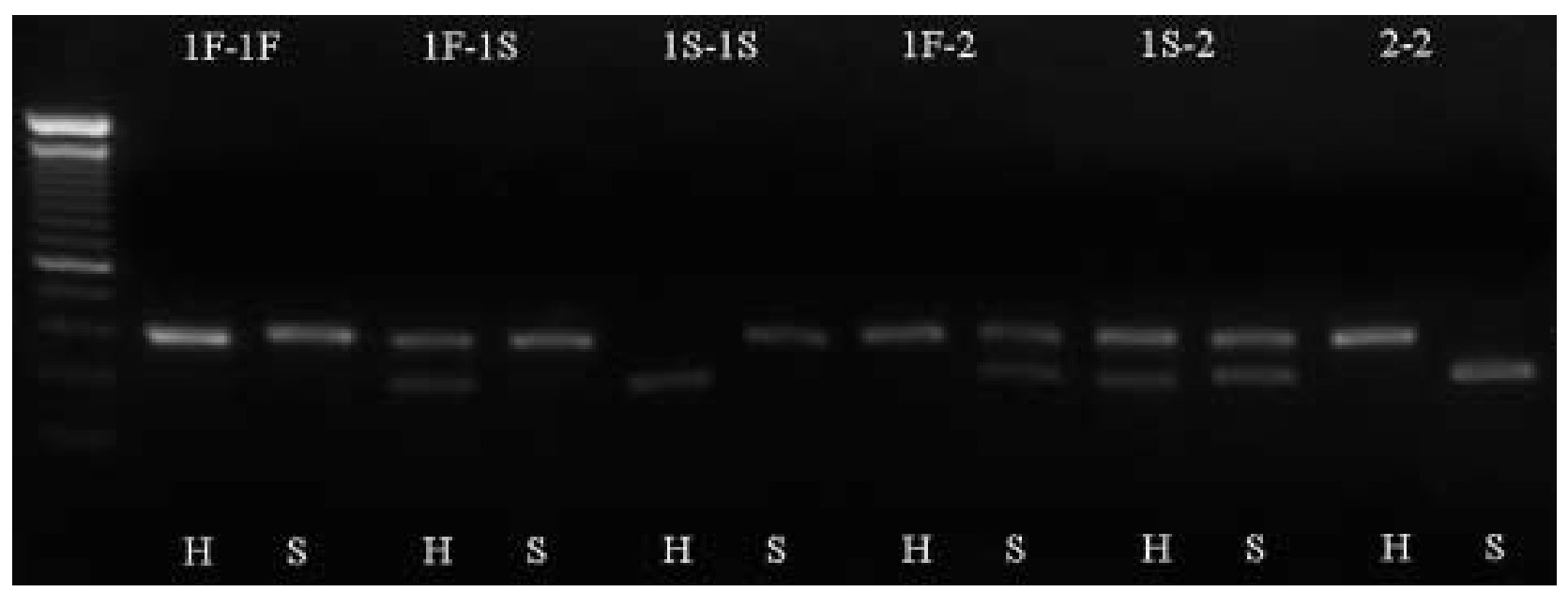

Fig. 1.-Representative electrophoresis gel with band visualization in the Gc-globulin polymorphism. All six genotypes are shown. The first lane is 100-1,000 base pairs (bp) deoxyribonucleic acid (DNA) ladder (the difference between each band is 100 bp). H: cut by Hae III; S: cut by Sty I; 1F: GC*1F; 1S: GC*1S; 2 : GC*2.

$60.3 \pm 13.8$; distribution of sex (male/female) was $60 / 3$ versus $45 / 37$; and $\mathrm{FEV} 1 / \mathrm{FVC}$ of pulmonary function tests was $44.3 \pm 12.7 \%$ versus $56.4 \pm 14.1 \%$, respectively. Other results of the pulmonary function tests of the COPD group were as follows: RV was $2.56 \pm 0.11 \mathrm{~L}$, TLC was $5.67 \pm 0.89 \mathrm{~L}, \mathrm{RV} / \mathrm{TLC}$ was $45.5 \pm 9.2 \%, D \mathrm{~L}, \mathrm{CO}$ was $11.0 \pm 3.4 \mathrm{~mL} \cdot \mathrm{min}^{-1} \cdot \mathrm{mmHg}^{-1}$, and $\% D \mathrm{~L}$, CO was $49.2 \pm 14.0 \%$. The average titre of cold haemagglutinin of the DPB group was $28.7 \pm 2.2$. Smoking history (pack-yrs) of the COPD subjects was $102.2 \pm 40.4$, and that of the control subjects was unknown because they were anonymous donors. The basic characteristics of COPD subjects are summarized in table 1.

The distribution of the six major genotypes of Gc-globulin in the control, COPD and DPB groups are presented in table 2 . The proportion of $\mathrm{GC}^{*} 1 \mathrm{~F}$ homozygotes was significantly higher in the COPD patients than in the control subjects (COPD 36.5\% versus control $20.7 \%, \mathrm{p}=0.0351$ ), and the odds ratio
(OR) for $\mathrm{GC}^{*} 1 \mathrm{~F}$ homozygotes versus all other genotypes was $2.2(95 \%$ CI 1.1-4.6) for the COPD group. The proportion with $\mathrm{GC}^{*} 1 \mathrm{~F}$ was also significantly higher in the COPD patients (COPD $85.7 \%$ versus control $75.6 \%, \mathrm{p}=0.0357)$, and the OR was $1.9(95 \%$ CI 1.2-3.2). In addition, the lung function parameters (FEV1/FVC, RV, TLC, RV/TLC, DL,CO, and $\% D \mathrm{~L}, \mathrm{CO})$ and smoking history between $\mathrm{GC}^{*} 1 \mathrm{~F}$ homozygotes and those of the other genotypes were compared. However, no correlation between these parameters and $\mathrm{GC}^{*} 1 \mathrm{~F}$ (data not shown) was detected.

The frequency of the three alleles is shown in table 3. In the control group, the frequency of the $\mathrm{GC}^{*} 1 \mathrm{~F}, \mathrm{GC}^{*} 1 \mathrm{~S}$, and $\mathrm{GC}^{*} 2$ allele was $0.48,0.27$, and 0.24 , respectively. The present results, differing from those of Caucasians (frequency of $\mathrm{GC}^{*} 1 \mathrm{~F}, \mathrm{GC}^{*} 1 \mathrm{~S}$, $\mathrm{GC}^{*} 2 ; 0.19,0.53,0.28$, respectively) [18], agree with those obtained in a previous study of a Japanese population [21]. Because the distribution of the sex

Table 1. - Basic characteristics of chronic obstructive pulmonary disease subjects

\begin{tabular}{|c|c|c|c|c|c|c|}
\hline $\begin{array}{l}\text { Smoking history } \\
\text { (pack-yrs) }\end{array}$ & FEV $1 / \mathrm{FVC} \%$ & $\begin{array}{c}\mathrm{RV} \\
\mathrm{L}\end{array}$ & $\begin{array}{c}\text { TLC } \\
\text { L }\end{array}$ & $\underset{\%}{\mathrm{RV} / \mathrm{TLC}}$ & $\begin{array}{c}D \mathrm{~L}, \mathrm{CO} \\
\mathrm{mL} \cdot \mathrm{min}^{-1} \cdot \mathrm{mmHg}^{-1}\end{array}$ & $\% D \mathrm{~L}, \mathrm{CO}$ \\
\hline $102.2 \pm 40.4$ & $44.3 \pm 12.7$ & $2.56 \pm 0.11$ & $5.67 \pm 0.89$ & $45.5 \pm 9.2$ & $11.0 \pm 3.4$ & $49.2 \pm 14.0$ \\
\hline
\end{tabular}

Data are presented as mean \pm SEM. FEV1: forced expiratory volume in one second; FVC: forced vital capacity; RV: residual volume; TLC: total lung capacity; $D$ L,CO: diffusing capacity of the lung for carbon monoxide $(\mathrm{mmHg} \times 0.133=\mathrm{kPa})$.

Table 2. - Distribution of the six major genotypes of Gc-globulin in the control, chronic obstructive pulmonary disease (COPD), and diffuse panbronchiolitis (DPB) groups

\begin{tabular}{llllllll}
\hline Groups & \multicolumn{7}{c}{ Six major genotypes } \\
\cline { 2 - 7 } & $1 \mathrm{~F}-1 \mathrm{~F}$ & $1 \mathrm{~F}-1 \mathrm{~S}$ & $1 \mathrm{~S}-1 \mathrm{~S}$ & $2-1 \mathrm{~F}$ & $2-1 \mathrm{~S}$ & $2-2$ & $1 \mathrm{~F}(+)$ \\
\hline Control & $17(20.7)^{\#}$ & $27(32.9)$ & $5(6.1)$ & $18(22.0)$ & $8(9.8)$ & $7(8.5)$ & $62(75.6)$ \\
COPD & $23(36.5)^{\#}$ & $15(23.8)$ & $1(1.6)$ & $16(25.4)$ & $6(9.5)$ & $2(3.2)$ & $54(85.7)$ \\
DPB & $16(19.5)$ & $18(22.0)$ & $9(11.0)$ & $21(25.6)$ & $11(13.4)$ & $7(8.5)$ & $55(67.1)$ \\
\hline
\end{tabular}

Data are presented as number of individuals (\% of group). $1 \mathrm{~F}$ : GC*1F; $1 \mathrm{~S}$ : $\mathrm{GC}^{*} 1 \mathrm{~S} ; 2$ : $\mathrm{GC} * 2 ; 1 \mathrm{~F}(+)$ : subjects with $\mathrm{GC} * 1 \mathrm{~F}$ allele. ${ }^{\#}$ : $p=0.0351 ;{ }^{\bullet}: \mathrm{p}=0.0357$. 
Table 3. - Frequency of the three alleles of Gc-globulin

\begin{tabular}{lccc}
\hline Groups & \multicolumn{3}{c}{ Allele frequency } \\
\cline { 2 - 4 } & $1 \mathrm{~F}$ & $1 \mathrm{~S}$ & 2 \\
\hline Control & 0.48 & 0.27 & 0.24 \\
Male & 0.49 & 0.30 & 0.21 \\
Female & 0.48 & 0.25 & 0.28 \\
COPD & 0.61 & 0.18 & 0.21 \\
DPB & 0.43 & 0.29 & 0.28 \\
\hline
\end{tabular}

COPD: chronic obstructive pulmonary disease; DPB: diffuse panbronchiolitis; 1F: GC*1F; 1S: GC*1S; 2: GC*2.

was different between the COPD (male/female, 60/3) and the control subjects (male/female, 42/40), the allele frequency between the male and female control subjects was compared. As shown in table 3, the difference of the allele frequency between these two groups was not statistically significant $(\mathrm{p}=0.99)$. Therefore, it was reasonable to consider that the difference of sex could not influence the distribution of this genetic variation.

The authors did not find any association between distribution of the genotype and frequency of the three polymorphic alleles of Gc-globulin and DPB (tables 2 and 3 ).

\section{Discussion}

COPD and DPB are diseases characterized by chronic airflow limitation. Although the aetiology of these diseases is still under investigation, neutrophils have commonly been assumed to play a key role in their pathogenesis. Therefore, variation in genes with a function related to chemotaxis of neutrophils may confer a risk for the development of COPD and DPB. Since Gc-globulin has been reported to be associated with chemotaxis of neutrophils [13] and monocytes [14], the authors investigated the association between genetic variation that codes the 416th and 420th amino acid of Gc-globulin and susceptibility to COPD and DPB.

This study showed that the proportion of COPD patients with the $\mathrm{GC}^{*} 1 \mathrm{~F}$ allele was significantly higher than that of control subjects with this allele. However, there was no difference in the allele frequency and distribution of genotypes of the Gc-globulin between the DPB and control groups.

Chronic tobacco smoking is the major risk factor for the development of COPD. However, only a small proportion of smokers develop airway limitation. The genetic factors are supposed to be related to this individual susceptibility. Recently, genetic polymorphism of cytochrome P450 [22], microsomal epoxide hydrolase [23], glutathione S-transferase M1 [24], tumour necrosis factor- $\alpha$ [25], and glutathione S-transferase P1 [26] were suggested to have an association with emphysema or COPD.

According to the results of this study, it is assumed that a genotype with the GC*1F allele might be one of the risk factors for COPD. Previously, the association between this genetic variation and COPD was reported by several studies. KUEPPERs et al. [16] have reported a lower frequency for the $\mathrm{GC}^{*} 2 / \mathrm{GC} * 2$ genotype in COPD (relative risk=0.2), and HoRNE et al. [17] have confirmed this data. However, in another study by KAUFFMANN et al. [19], no association was found, and the role of the GC*2 allele of Gc-globulin in the pathogenesis of COPD has remained controversial. All of these studies [16, 17, 19] were performed at a serological level. A recent study by ScHELLENBERG et al. [18] was performed at a DNA level and reported the protective effect of the $\mathrm{GC}^{*} 2 / \mathrm{GC}^{*} 2$ genotype for $\mathrm{COPD}(\mathrm{OR}=0.17)$. However, the present study could not confirm this effect. It is possible that racial differences might have caused this discrepancy between results $[18,21]$, however, this remains to be elucidated.

These reports showed the common finding that the frequency of the $\mathrm{GC}^{*} 1 \mathrm{~F}$ allele was higher in the COPD group [16-19], and one report also suggested that the homozygous $\mathrm{GC}^{*} 1 \mathrm{~F}$ allele increased the risk of developing COPD [17], which was compatible with the present results.

The difference of the function of Gc-globulin between the $\mathrm{GC}^{*} 1 \mathrm{~F}$ allele and the other alleles has not yet been elucidated. The different genotypes of Gc-globulin are known to have post-translational carbohydrate differences [27]. Gc1 and Gc2 were reported to have different pathways of deglycosylation [28], and the deglycosylated form of Gc-globulin has been shown to be a potent macrophage-activating factor [27]. Therefore, in addition to the possibility that Gc-globulin polymorphism modulates neutrophil and monocyte chemotaxis, the differences in deglycosylated forms of Gc1f, Gc1s, and Gc2 may effect activation of macrophages, whose metalloproteinase was recently reported to play a key role in the pathogenesis of emphysema [29].

Although the $\mathrm{GC}^{*} 2$ allele has been reported to be associated with the chemotactic activity of neutrophils [13], this was not ascertained in a recent study [18], and the present authors found no association between the genotype with the $\mathrm{GC}^{*} 2$ allele and the susceptibility to COPD.

In conclusion, it is suggested that the genotype the $\mathrm{GC}^{*} 1 \mathrm{~F}$ allele of Gc-globulin might be one of the risk factors for chronic obstructive pulmonary disease. However, no association between this polymorphism of Gc-globulin and susceptibility to diffuse panbronchiolitis was found in the present study.

\footnotetext{
Acknowledgements. The authors sincerely thank K. Nakata and Y. Taguchi for their help.
}

\section{References}

1. Murray CJL, Lopez AD. Evidence-based health policy-lessons from the global burden of disease study. Science 1996; 274: 740-743.

2. Fletcher C, Peto R. The natural history of chronic airflow obstruction. BMJ 1977; 1: 1645-1648. 
3. Sandford AJ, Weir TD, Pare PD. Genetic risk factors for chronic obstructive pulmonary disease. Eur Respir $J$ 1997; 10: 1380-1391.

4. Silverman EK, Chapman HA, Drazen JM, et al. Genetic epidemiology of severe, early-onset chronic obstructive pulmonary disease. Risk to relatives for airflow obstruction and chronic bronchitis. $\mathrm{Am}$ J Respir Crit Care Med 1998; 157: 1770-1778.

5. Robin ED. Summary: symposium on pulmonary emphysema and proteolysis. In: Mittman C, ed. Pulmonary Emphysema and Proteolysis. New York, Academic Press, 1972; pp. 527-537.

6. Homma H, Yamanaka A, Tanimoto S, et al. Diffuse panbronchiolitis: a disease of the transitional zone of the lung. Chest 1983; 83: 63-69.

7. Kim YW, Han SK, Shim YS, et al. The first report of diffuse panbronchiolitis in Korea: five case reports. Intern Med 1992; 31: 695-701.

8. Hu H, Liu YN. The first case of diffuse panbronchiolitis reported in China. Therap Res 1996; 17: 339-344.

9. Keicho N, Tokunaga K, Nakata K, et al. Contribution of HLA genes to genetic predisposition in diffuse panbronchiolitis. Am J Respir Crit Care Med 1998; 158: 846-850.

10. Ichikawa $\mathrm{Y}$, Ninomiya $\mathrm{H}$, Koga $\mathrm{H}$, et al. Erythromycin reduces neutrophils and neutrophil-derived elastolytic-like activity in the lower respiratory tract of bronchiolitis patients. Am Rev Respir Dis 1992; 146: 196-203.

11. Kadota J, Sakito O, Kohno S, et al. A mechanism of erythromycin treatment in patients with diffuse panbronchiolitis. Am Rev Respir Dis 1993; 147: 153-159.

12. Oda H, Kadota J, Kohno S, Hara K. Erythromycin inhibits neutrophil chemotaxis in bronchoalveoli of diffuse panbronchiolitis. Chest 1994; 106: 1116-1123.

13. Kew RR, Webster RO. Gc-globulin (vitamin D-binding protein) enhances the neutrophil chemotactic activity of C5a and C5a des Arg. J Clin Invest 1988; 82: 364-369.

14. Piquette CA, Robinson-Hill R, Webster RO. Human monocyte chemotaxis to complement-derived chemotaxins is enhanced by Gc-globulin. J Leukoc Biol 1994; 55: 349-354.

15. Kamboh MI, Ferrell RE. Ethnic variation in vitamin D-binding protein $(\mathrm{Gc})$ : A review of isoelectric focusing studies in human populations. Hum Genet 1986; 72: 281-293.

16. Kueppers F, Miller RD, Gordon H, Hepper NG, Offord K. Familial prevalence of chronic obstructive pulmonary disease in a matched pair study. $\mathrm{Am} \mathrm{J} \mathrm{Med}$ 1977; 63: 337-342.

17. Horne SL, Cockcroft DW, Dosman JA. Possible protective effect against chronic obstructive airways disease by the GC2 allele. Hum Hered 1990; 40: 173176.

18. Schellenberg D, Pare PD, Weir TD, Spinelli JJ, Walker BA, Sandford AJ. Vitamin D binding protein variants and the risk of COPD. Am J Respir Crit Care Med 1998; 157: 957-961.

19. Kauffmann F, Kleisbauer JP, Cambon-De-Mouzon A, et al. Genetic markers in chronic airflow limitation: a genetic epidemiologic study. Am Rev Respir Dis 1983; 127: 263-269.

20. American Thoracic Society. Standards for the diagnosis and care of patients with chronic obstructive pulmonary disease. Am J Respir Crit Care Med 1995; 152: S77-S120.

21. Watanabe Y, Yamada S, Nagai A, et al. Japanese population DNA typing data for the loci LDLR, GYPA, HBGG, D7S8, and GC. J Forensic Sci 1997; 42: 911-913.

22. Cantlay AM, Lamb D, Gillooly M, et al. Association between the CYP1A1 gene polymorphism and susceptibility to emphysema and lung cancer. $J$ Clin Pathol Mol Pathol 1995; 48: M210-M214.

23. Smith CAD, Harrison DJ. Association between polymorphism in gene for microsomal epoxide hydrolase and susceptibility to emphysema. Lancet 1997; 350: 630-633.

24. Harrison DJ, Cantlay AM, Rae F, Lamb D, Smith CA. Frequency of glutathione S-transferase M1 deletion in smokers with emphysema and lung cancer. Human Exp Toxicol 1997; 17: 356-360.

25. Huang S-L, Su C-H, Chang S-C. Tumor necrosis factor-alpha gene polymorphism in chronic bronchitis. Am J Respir Crit Care Med 1997; 156: 1436-1439.

26. Ishii $\mathrm{T}$, Matsuse $\mathrm{T}$, Teramoto $\mathrm{S}$, et al. Glutathione S-transferase P1 (GSTP1) polymorphism in the patients with chronic obstructive pulmonary disease (COPD). Thorax 1999; 54: 693-696.

27. Svasti J, Kurosky A, Bennett A, Bowman BH. Molecular basis for the three major forms of human serum vitamin D binding protein (group-specific component). Biochemistry 1979; 18: 1611-1617.

28. Yamamoto N, Homma S. Vitamin D3 binding protein (group-specific component) is a precursor for the macrophage-activating signal factor from lysophosphatidylcholine-treated lymphocytes. Proc Natl Acad Sci USA 1991; 88: 8539-8543.

29. Hautamaki RD, Kobayashi DK, Senior RM, Shapiro S. Requirement for macrophage elastase for cigarette smoke-induced emphysema in mice. Science 1997; 277: 2002-2004. 